\title{
ENSEÑANZA DE LA FILOSOFÍA Y NUEVAS PRÁCTICAS FILOSÓFICAS
}

\author{
Miguel Ángel Gómez Mendoza* \\ Universidad Tecnológica de Pereira.
}

\section{Resumen}

Una de las inquietudes más grandes de la sociedad contemporánea, frente a sus incertidumbres y angustias, así como una de la preocupaciones de los profesores de filosofía ante un público cada vez más mediático, es la de cómo abordar la filosofía con medios más pedagógicos: cómo "didactizarla", volverla "filosofía práctica" y dialogal, no obstante, esta pretensión, ha sido también cuestionada por los docentes que consideran los riesgos de superficialidad, espontaneismo y "opinadera" de tales propuestas pedagógicas que desvirtúan la filosofía como disciplina. Esta es la problemática que este artículo pone en discusión.

Palabras clave: enseñanza de la filosofía; ciudadanía; filosofía para niños.

\begin{abstract}
Philosophy teaching and contemporary philosophical practice

One of the most disquieting concerns of contemporary society regarding its own uncertainties and anxieties, as well as the one of the central preoccupations of philosophy professors, confronted with students that are more and more imbued by the media, is how to approach philosophy with more pedagogical methods; how to make it more didactic, how to propose a philosophical and dialogical practice. Nevertheless, this pretension has also been criticized by teachers that consider the risks of reducing philosophy to the teaching of citizenship, or worse yet, to the shallowness, spontaneity and "opinion-giving" that such pedagogical proposals tend to produce, and which diminish the academic discipline of philosophy. This is the controversy that the present article examines.
\end{abstract}

Key words: Philosophy teaching, citizenship, philosophy for children.

\section{Concepciones o paradigmas de la enseñanza de la filosofía y nuevas prácticas de la enseñanza filosófica}

Como aprecia Pierre Sané (subdirector general para las ciencias sociales y humanas de la Unesco), en el prefacio del informe "La philosophie. Une Ecole de la Liberté. Enseignement de la philosophie et apprentissage du philosopher. Etat des lieux et regards pour l'avenir" (2007: XIV), un estado de la cuestión de la filosofía hoy se puede realizar cuando se esbozan cuatro facetas de

\footnotetext{
* Facultad de Educación. mgomez@utp.edu.co
} 
su enseñanza, con el fin de abarcar todos los niveles, que implican tanto la educación formal como la educación informal: (i) la filosofía y las jóvenes mentes, la edad del asombro -su enseñanza en los niveles preescolar y primaria ${ }^{1}$-; (ii) la filosofía en la edad del cuestionamiento su enseñanza en el nivel secundario-; (iii) la filosofía en el campo universitario -su enseñanza en el nivel universitario-; (iv) descubrir la filosofía de otra manera -su práctica en la ciudad o en la esfera de lo público- ${ }^{2}$.

La originalidad de este estudio de la Unesco se traduce en la identificación de cuestiones vivas, que interpelan de manera constante a los Estados, como a los profesores, los investigadores y los practicantes de la filosofía. Se puede destacar la cuestión de la educabilidad filosófica de la infancia con sus dimensiones psicológica, filosófica y sociológica; la importancia de las innovaciones en materia pedagógica; el papel fundamental del profesor y la cuestión de la formación de los formadores; la cuestión del retiro o del reemplazo de la enseñanza filosófica; las salidas u opciones profesionales; la necesidad de filosofar; e incluso el estatuto y la posición de la filosofía en nuestros días.

\subsection{El paradigma “organizador" y los enfoques de la enseñanza de la filosofía}

Si bien es posible determinar en términos nacionales, continentales o geográficos las prácticas y métodos de enseñanza de la filosofía en el mundo, como lo hace el informe mencionado, para los propósitos expositivos y argumentativos de esta comunicación, en primer lugar, apelamos a la "tipología" de los cinco paradigmas principales de la enseñanza de la filosofía y del filosofar, que

\footnotetext{
${ }^{1}$ Más adelante se encuentran las referencias al profesor Diego Antonio Pineda, de la Universidad Javeriana de Bogotá, sobre el nacimiento y aplicación de la filosofía para niños en Colombia.

${ }^{2}$ Según Oscar Brenifier: "Es difícil determinar y definir la actividad filosófica no académica. ¿Cómo llamarla? Filosofía informal, natural, popular, no institucional, extramuros... Todo depende obviamente de lo qué se entienda por filosofía. La disputa o querella entre la enseñanza de los sofistas y la mayéutica socrática inaugura esta disputa, consubstancial con la historia de la filosofía. Ambigüedad del doble término de sophia: la filosofía como transmisión de saber o la filosofía como aprendizaje de la sabiduría. Se encuentra igualmente en Kant la distinción entre una filosofía popular y una filosofía académica. Eterno debate, que se vuelve a encontrar, más recientemente, entre filósofos, para determinar si sí o no existe una filosofía no occidental, africana, china o hindú. Para los partidarios de la tesis "clásica" de inspiración heideggeriana, según la cual la filosofía nació específicamente en Grecia en la época clásica, una concepción amplia de la filosofía será no solamente rechazada, sino que corre el riesgo de generar un escándalo. Esta visión restrictiva de la filosofía es sin duda una de las razones por las cuales esta disciplina, hasta una fecha reciente, parece estar acantonada principalmente en el interior de los muros de la clase o en los recintos de las bibliotecas" (Unesco, 2007: 153). Este mismo autor nos ofrece información, testimonios ilustrativos, experiencias concretas que tienen lugar hoy en el campo del "filosofar de otra forma" en el capítulo IV del informe de la Unesco, al cual se ha hecho referencia ("Découvrir la philosophie autrement. La philosophie dans la Cité-“. ¿Filosofía en otra parte, filosofar de manera diferente?”) (2007:151-195). En el panorama de la pluralidad de las prácticas filosóficas "no académicas", es decir, las modalidades de "filosofía práctica", se encuentran: la consulta filosófica, el café filosófico, el taller de filosofía, el éxito de ciertas obras editoriales (El libro de Sofía de Jostein Gaarder; La potencia de existir. Manifiesto hedonista y el Antimanual de filosofía, de Michel Onffray; Más Platón menos Prozac de Lou Marinoff, y el reciente The Simpsons and philosophy: the D'oh of Homer donde 20 filósofos, liderados por William Ervin de la Universidad de Pensilvania, reflexionan y escriben ensayos sobre esta familia y su entorno en la desternillante ciudad de Springfield, que la editorial española Blackie publicara en el último trimestre de este año con el título Los Simpsons y la filosofía; la filosofía con los niños fuera de la escuela; el filosofo en la empresa; la filosofía en medios difíciles o en medios con población altamente vulnerable, como se dice en Colombia.
} 
coexisten en el espacio didáctico europeo y que permiten ver sus grandes tendencias ${ }^{3}$ (Tozzi, 2007, 2008; Unesco: 83-85).

El interés del trabajo de Michel Tozzi reside en el objetivo de determinar las prácticas pedagógicas que permiten constituir la filosofía como una materia aparte, es decir, llevar a cabo el paso de un conjunto de contenidos filosóficos a la manera como la filosofía, como disciplina cultural, históricamente determinada y universitariamente situada, se didactiza en la enseñanza secundaria (y ahora en preescolar y primaria), es decir, se convierte en una materia escolar.

La filosofía -campo esencial de la cultura por su historia, sus doctrinas y sus métodos de pensamiento-, cuando se convierte en una materia enseñada en el sistema educativo, debe didactizarse, es decir, tomar una forma que pueda dar lugar a los aprendizajes accesibles a los alumnos. Los didactas de esta disciplina denominan o definen el "paradigma organizador" de una disciplina o su "matriz disciplinar" como la manera en que ella se organiza para ser enseñada, que implica elementos como: finalidades y objetivos fijados o establecidos, presencia en tal o cual nivel o modalidad académica, la enseñanza, horarios, objetos, tareas y soportes propuestos, métodos de enseñanza y de aprendizaje, contenidos de los programas (nociones, cuestiones, preguntas, textos, manuales o libros de texto), formas de evaluación y tipos de examen. La misma disciplina filosófica puede de esta manera adquirir diferentes formas o facetas según los tiempos y los lugares en que se enseñe; esto es, "paradigmas".

En este contexto, para Tozzi (2008, 2006, 2005, 2005a), la enseñanza de la filosofía se puede describir y comprender a partir de un "paradigma organizador" en un conjunto tipológico de paradigmas. Así tendríamos los siguientes tipos de paradigmas:

a. Doctrinal o dogmático-ideológico: la filosofía oficial de la Iglesia (tomismo) en la edad media, "sirvienta de la teología"; el marxismo-leninismo en los países ex comunistas o del socialismo real, o la enseñanza oficial de la filosofía en la España franquista; la filosofía se presenta como una respuesta organizada y coherente a las cuestiones fundamentales de la humanidad.

b. Histórico-patrimonial: la filosofía aparece aquí como una forma histórica mayor de la cultura, la manera como la humanidad, para responder a las preguntas que ella se plantea sobre su condición, ha pasado del mito (mito que intenta explicar bajo la forma narrativa, metafórica) al logos (el discurso racional, que la filosofía comparte con la ciencia). La humanidad elaboró entonces en la historia visiones de mundo, especies de sistemas explicativos, de la relación del hombre con el cosmos, con el otro, consigo mismo; ella es así una historia de sus tentativas para

\footnotetext{
${ }^{3}$ El trabajo de Michel Tozzi viene desarrollándose desde 1992 (por ejemplo con su tesis de doctorado: Vers une didactique du philosopher), mediante la publicación de numerosos libros y artículos: una "didactización del aprendizaje del filosofar" (du philosopher) desde el ámbito de la filosofía -y no tanto desde la perspectiva de la pedagogía-. Esto es, una fundamentación filosófica de la didáctica del filosofar ( $d u$ philosopher), haciendo hincapié en el verbo, en el proceso, en la actividad de examinar algo como filósofo, o discurrir acerca de ello con razones filosóficas), más que de la filosofía ("philosophie", el sustantivo, el resultado final, la disciplina). Concibe la actividad filosófica como una práctica que comprende tres capacidades o estadios: la problematización, la conceptualización y la argumentación que engloba la lectura, la escritura y la discusión filosófica (Cf.: Tozzi, Michel (2005) Penser par soi-même. Initiation à la philosophie. Lyon: Chronique Sociale. 215 páginas. Cf.: Página Web denominada philotozzi: http://www.philotozzi.com.
} 
comprender y actuar sabiamente. Su didactización es una especie de "historia de las ideas", con sus momentos fuertes y determinantes de cada época intelectual. Se parte de la historia de las ideas presocráticas a nuestros días; se enseña, sobre todo, contenidos ideológicos o doctrinales, patrimoniales; por ejemplo, la mayéutica socrática, la idea platónica, la retórica aristotélica, el coraje estoico, la teología tomista, la duda cartesiana, el imperativo kantiano, la dialéctica hegeliana, la plusvalía marxiana, la sospecha nietzscheana, el inconsciente freudiano, la duración bergsoniana, la descripción husserliana, el Dasein heideggeriano, etc.

c. Problemático o problematizador: paradigma en el que las nociones y los textos filosóficos tienen su lugar, ante todo, por su relación con los problemas filosóficos. Aquí interesa "pensar por sí mismo" en los procesos de pensamiento del filosofar, pensar problemas estrechamente relacionados en la enseñanza institucional o "tradicional" de la filosofía, con el curso del profesor como "obra", con los textos filosóficos como ejemplos y modelos de pensamiento, con lo escrito como disertación, como forma obligada de aprendizaje filosófico. Todo esto se hace para que los alumnos puedan comenzar a construir en sus cabezas un pensamiento propio, que se convertirá progresivamente en su visión del mundo. No se trata ni de exponer una historia de las ideas, ya que los conceptos, las doctrinas, el curso no están para provocar el pensamiento de los alumnos y menos aún para imponer una doctrina oficial, puesto que es preciso un encaminamiento personal del alumno. Este es quizá el caso francés, donde el aprendizaje de la cultura filosófica debe estar investido por la exposición de problemas y por el ensayo metódico de sus formulaciones y de sus soluciones posibles, y donde la enseñanza de la filosofía en el último año de bachillerato tiene por objetivo favorecer el acceso de cada alumno al ejercicio reflexivo del juicio, al desarrollo del sentido de la responsabilidad intelectual, a formar espíritus autónomos, capaces de elaborar una conciencia crítica del mundo contemporáneo.

d. Democrático-discutidor: su objetivo es también problematizador, como el anterior, pero su originalidad reside en intentar articular el objetivo de aprender a pensar por sí mismo con una pretensión democrática. La enseñanza filosófica se concibe, de esta forma, en una perspectiva de educación para la ciudadanía o la democracia, sin que esta enseñanza sea necesariamente subordinada. La idea es que la democracia, como régimen político, tiene necesidad, para profundizarse, de ciudadanos reflexivos, es decir, capaces de tener un espíritu crítico, resistente a las desviaciones siempre posibles de la democracia: la doxología, el reino o dictadura de la opinión y del número, la sofística, convencer por todos los medios, la demagogia, etc.; aquí la democracia está consubstancialmente asociada al debate, a la discusión, que aseguran un derecho de expresión y una pluralidad de opiniones; se trata de consolidar reflexivamente el debate democrático.

e. Praxeológico-ético: en los cursos de moral y ética; el objetivo es orientarse reflexivamente en la acción, por un esfuerzo de clarificación y jerarquización de los valores. En este paradigma la atención se centra en la praxis, la acción, se trata de aprender a actuar y no solamente a pensar, con el fin de vivir bien, es decir, conforme a los valores. Filosofar es adoptar con conocimiento de causa, una cierta conducta ética. Focalizar la enseñanza de la filosofía sobre el mero aprendizaje del pensamiento sería amputar la disciplina de una dimensión fundamental, aquella que pretende, como decía Marx en sus Tesis sobre Feuerbach, no solamente "interpretar el mundo sino transformarlo". Una forma histórica de este paradigma es la sabiduría de los filósofos predicadores en la antigüedad. El pensamiento no se basta a sí mismo para aclarar nuestra comprensión del mundo; él apunta a un cierto tipo de vida "buena", conforme a la razón y 
conducente a la felicidad; ya sea por el placer mesurado, el epicureismo o por el ejercicio de la virtud, el estoicismo. El filósofo no es en este paradigma solamente un maestro del pensamiento, sino un maestro de la acción. Se encuentra una concepción modernizada de este paradigma en algunas versiones de cursos de moral o de ética. En estos, se parte de la reflexión, por ejemplo, a partir de dilemas morales, para aprender a clarificar y a jerarquizar los valores con el objetivo de actuar éticamente a sabiendas y en el momento oportuno, sin que estos valores sean por lo tanto impuestos, ya que ellos surgen del libre examen. La noción de compromiso es aquí central, en su dimensión individual y colectiva.

\subsection{Crisis y proposiciones en la enseñanza de la filosofía}

Esta breve relación de paradigmas de la enseñanza de la filosofía y del enseñar filosófico permitiría afirmar que la educación comparada muestra que se puede enseñar la filosofía en el sistema educativo de diferentes maneras: enseñando en el bachillerato la historia de las ideas, el patrimonio filosófico occidental desde los presocráticos hasta Heidegger, como en Italia, donde el docente es al mismo tiempo profesor de historia y de filosofía (Sánchez: 1996); transmitiendo una ideología oficial, como el marxismo-leninismo en la antigua Unión Soviética; educando el razonamiento a través de la clarificación y jerarquización de los valores con el fin de actuar éticamente, como desarrollan en Bélgica (Dortu, 2004) los profesores de moral; o aprendiendo a reflexionar sobre un conjunto de problemas y nociones, como en Francia (Tozzi, 2007). En el caso de nuestro País: como educación filosófica (Pineda: 2004) y como disciplina universitaria en una universidad pedagógica (Sarmiento, 2004); o en el caso español contemporáneo, donde la filosofía se justifica en la enseñanza secundaria como una introducción histórica y filosófica a los problemas filosóficos y herramienta conceptual insustituible para que los alumnos aprendan a ejercitarse, y es una responsabilidad directa e inmediata de la filosofía el ejercicio crítico de la razón; objetivo este que se logra con la elaboración de unidades didácticas que comportan contenidos conceptuales, procedimientales y actitudinales, con sus respectivas actividades y modalidades de evaluación (Cifuentes, 1997).

Igualmente, la historia de la enseñanza de la filosofía en Francia (Tozzi, 2007; Poucet, 1996, 2004, Serrat, 2001, Levent, 2001) nos muestra que el paradigma organizador o "matriz disciplinar" tradicional de su enseñanza se estabiliza a finales del siglo XIX, restringido al último curso de bachillerato de los institutos de élite de aquella época (en 1900, sólo uno de cada cien jóvenes adquiría el título de bachiller). Este tipo de enseñanza se fundamenta sobre tres pilares básicos: a) la clase magistral del profesor de filosofía, concebida como una "obra" ejemplar de pensamiento en acción; b) los grandes textos de filosofía, y c) la disertación y comentario de texto como modelos acabados de pensamiento para aprender filosofía o aprender a filosofar.

Ahora bien, el paradigma "organizador" o matriz de la enseñanza de la filosofía, pese a sus variaciones contextuales nacionales expuestas en términos de paradigmas, parece haber evolucionado muy poco: los alumnos, generalmente motivados a principio de curso con la esperanza de poder expresarse sobre cuestiones existenciales, pierden rápidamente el interés frente a la aridez de la clase magistral, la dificultad de los textos filosóficos y la baja calificación de las disertaciones y los resultados de los comentarios de textos filosóficos. Los profesores luchan con los "nuevos bachilleres", que carecen de los hábitos lingüísticos y culturales para mantener sus exigencias intelectuales $-\mathrm{y}$ a menudo incluso hasta la atención de sus propios alumnos-, mientras que, a pesar de este cambio de circunstancias, siguen privilegiando la 
posición frontal del discurso de alto nivel del profesor, e incorporando muy pocas situaciones activas como el trabajo en grupo o las discusiones.

Existen, según Michel Tozzi (2007: 209), razones más generales para hablar de cierta crisis de la enseñanza vinculada con la evolución social y escolar, que dificultan cada vez más la relación de numerosos alumnos con el conocimiento y con las normas legales. Para el autor, el discurso antipedagógico dominante entre los representantes mayoritarios de la representación filosófica tiene también su parte de responsabilidad: formación inicial y continua excesivamente académica, subestimando la dimensión pedagógica de su profesión y la importancia de una cultura de la mutualización ${ }^{4}$ de las prácticas profesionales (¿cuántos conceptos y cuáles deben ser enseñados?), discurso y práctica que se resiste al cuestionamiento de los métodos de enseñanza; oposición a una reflexión didáctica que tome en cuenta la apuesta y el desafío de una enseñanza filosófica de masas, que forzaría una evolución del paradigma clásico u organizador, sentimiento de una disciplina aparte y en ruptura con las otras, cierta petulancia y autosuficiencia disciplinaria (la filosofía como disciplina sabia y aristocrática frente a otras menos sabias, instrumentales y plebeyas), tendencia a transferir las dificultades de desempeño oral y escrito de los estudiantes a los niveles inferiores del sistema educativo, en lugar de abordarlas de frente y desde otras perspectivas.

Se han sugerido diversas propuestas para intentar desbloquear esta situación: detallar aún más el currículo, para reducir la extensión de los problemas que se presentan en los exámenes; clarificar e incluir en el currículo oficial ${ }^{5}$ las competencias reflexivas que se requieren y los criterios de su evaluación, para incrementar su visibilidad más radicalmente, puesto que aquí se cuestiona el propio paradigma; desarrollar métodos de enseñanza más activos, además de la clase magistral (valorar la argumentación oral de los alumnos, no solamente la clase dialogada o la exposición, sino también el trabajo en grupo y la discusión en toda la clase); diversificar los ejercicios que se proponen, el trabajo interdisciplinario, las formas de escritura filosófica, el tipo de examen; en el campo de la formación docente, además de la legítima enseñanza académica sobre los contenidos, desarrollar la dimensión didáctica de la enseñanza de la filosofía, los conocimientos pedagógicos sobre la dinámica y la gestión del grupo-clase, las teorías del aprendizaje (socioconstructivismo) $)^{6}$, la evaluación, los actuales métodos de análisis de prácticas, y

\footnotetext{
${ }^{4}$ Se utiliza este termino "mutualización" procedente en principio del ámbito empresarial y que se ha extendido a otros ámbitos y disciplinas, como por ejemplo, al ámbito social y educativo, que implica potenciar una cultura en la que se incremente la colaboración y la cooperación entre profesionales del mismo campo (en el caso que nos ocupa, por docentes de la misma área, del mismo nivel o etapa, de la misma institución educativa), normalmente a través de las TIC, institucionalizando esta colaboración de algún modo.

${ }^{5}$ Aquí cabria hacer referencia al proceso de elaboración de los "Lineamientos curriculares para la enseñanza y el aprendizaje de la filosofía" en nuestro país, mediante el convenio suscrito entre el Ministerio de Educación Nacional y la Universidad Pontificia Bolivariana de Medellín (2006), uno de cuyos documentos circuló como borrador en noviembre de 2007; 113 páginas.

6 «D'un point de vue psychologique, le constructivisme met l'accent sur le rôle central de l'apprenant dans le processus d'appropriation des savoirs et sur la prise en compte de la logique des apprentissages qui n'est pas réductible à la logique des savoirs. Alors que les approches cognitives de l'apprentissage s'efforcent de mettre en évidence les opérations cognitives ou les processus mentaux par lesquels s'effectue l'apprentissage, les approches socio-cognitives se caractérisent par l'idée que, si tout apprentissage fait effectivement intervenir l'activité cognitive de celui qui apprend, il ne s'effectue pas dans un vide social (Doise et Mugny, 1981). Cela conduit à insister davantage sur le caractère contextualisé des apprentissages et sur le rôle central des interactions sociales, en
} 
fundamentalmente adelantar la edad de su enseñanza, estableciendo una progresividad en el aprendizaje de la filosofía y del filosofar.

Ahora bien, como se sugiere en los capítulos I, II y IV del informe de la Unesco (2007), cuando se ocupa de la enseñanza y el aprendizaje del filosofar en los niveles preescolar, primaria y secundaria, incluyendo el "descubrimiento" de otras formas de la filosofía, sería necesario actuar sobre las causas específicas de la crisis actual de la enseñanza y del aprendizaje de la actividad filosófica; es necesario promover prácticas que articulen la exigencia de la disciplina y que tengan en cuenta el nuevo contexto de las instituciones educativas masificadas, crear institutos de investigación sobre la enseñanza de la filosofía, animar y difundir las prácticas innovadoras que ensayan vías inéditas para "aprender a filosofar", abiertas a experiencias diferentes y a la reflexión sobre nuevas formas de filosofía ${ }^{7}$. Una de estas prácticas es precisamente la discusión en educación, y en este caso particular, la discusión de tipo filosófico.

\section{Discusión en educación y la discusión de tipo filosófico}

Para Tozzi (2004: 9-19), la discusión permite el examen en un grupo de un problema pendiente, para intentar formularlo y avanzar colectivamente hacia su solución, articulando diferentes dimensiones: lingüísticas y lenguajes verbal y no verbal, y compromete una participación decidida de los actores en términos afectivos y cognitivos.

Como práctica social reglada de la discusión, cuando adquiere, por ejemplo, la forma de "confrontación de las opiniones", ha sido amplificada por el desarrollo de los medios de comunicación y se ha difundido por la sociedad global. La exigencia democrática, inseparable de la obligación de discutir para determinar el bien común, parece desarrollarse con el aumento del nivel de educación de la población, y con una creciente complejidad en la sociedad, que

particulier le rôle médiateur que peut exercer un sujet plus compétent auprès d'un sujet moins compétent, dans le processus d'acquisition de connaissances nouvelles. Dans cette deuxième perspective, si l'apprentissage est bien le résultat d'une construction interne, effectuée par l'apprenant, celle-ci n'est pas seulement individuelle et cognitive, mais sociale et affective $»$. (Jonnaert, 2004: p. 71).

7 Cuando se habla de "nuevas formas de filosofía" se podría hacer referencia ilustrativa a la llamada "filosofía práctica": "Naturalmente, cuando se hace referencia al término Philosophical Practice, que podríamos traducir en español como "Filosofía práctica", el público más o menos especializado suele entender que estamos hablando fundamentalmente de Philosophical Counseling, esto es de Orientación filosófica. De hecho, cuando Achenbach introdujo este neologismo en lengua alemana se estaba refiriendo exclusivamente a esta modalidad de práctica filosófica. Reducir el conjunto de prácticas filosóficas que engloba la disciplina de la Filosofía Práctica exclusivamente al área del Asesoramiento filosófico constituye, a mi juicio, una visión limitada y restrictiva de lo que es realmente este movimiento y de su verdadero alcance [... [ Consideramos pues, que el campo de la filosofía práctica se puede distribuir en cuatro áreas o ámbitos de actuación: 1) El ámbito terapéutico, que corresponde al área de la Orientación filosófica; 2) El ámbito lúdico, desarrollado principalmente a través de (a) cafés filosóficos, (b) talleres de filosofía, (c) diálogos socráticos; 3) El ámbito formativo, que se desarrolla en las organizaciones, ya sean estas (1) instituciones públicas, (2) empresas privadas o (3) ONG, y, por último, (4) El ámbito mediático, a través de la labor divulgativa en los distintos mass media. Por consiguiente, la formación del futuro filósofo práctico no debería limitarse únicamente a proporcionar los instrumentos teóricos y prácticos pertenecientes al trabajo del orientador filosófico, sino que debería incluir también de forma extensa y no solo marginalmente, las diferentes aportaciones teóricas y las diversas propuestas metodológicas de los otros ámbitos. Sobre todo, si tenemos en cuenta que este campo de actuación es mucho más rentable económicamente que el de la Orientación Filosófica y posee muchas más potencialidades de desarrollo profesional" (Arnaiz, 2007: 1-2). 
multiplica los niveles, las instancias y exigencias de la discusión, y que además debe tratar de articular la diversidad de los grupos de interés.

La discusión se enraíza públicamente en el sentimiento de insatisfacción frente a la representación política y a la crisis del lugar del ciudadano en el espacio público. El aumento del individualismo y el "debilitamiento de la institución" que preformaba cada uno de los hábitos de los roles funcionales llevan a recurrir a la fuerza física o a un refuerzo legislativo o a una discusión para resolver los conflictos o regular las disfunciones crecientes de la comunicación. El individuo es conducido en los contextos colectivos y en los disensos sobre valores comunes a negociar y a procesos contractuales que implican muchas veces sus relaciones privadas, para tejer así la coexistencia sobre la base de intereses colectivos presentes en la sociedad.

La discusión está a la orden del día. Se discute, y mucho, sobre diversos aspectos de la vida diaria: se discute y negocia con la pareja, a partir del hecho sociológico y cultural en el que la mujer es conducida por la corriente secular de la emancipación financiera y de la autonomía personal; con los niños y con los jóvenes, sobre la selección de las distracciones, las actividades, la orientación, los estudios y el futuro profesional, la profesión, sobre las horas de salida, la compra de un medio de desplazamiento, la cantidad de dinero de bolsillo, etc.; se discute también con los amigos, los colegas de trabajo; entre patrones y asalariados, no solamente en las relaciones sindicales clásicas, que entremeten relaciones de fuerza y de negociación, en el marco de la "gestión participativa de los recursos humanos", del trabajo en equipo, etc. El sistema educativo no escapa a esta dinámica; la discusión también está presente en él; veamos algunos indicadores:

a. En la vida escolar (ejemplo la formación de los alumnos delegados o representantes de clase).

b. En la vida de la clase: se estimula en el nivel de la básica primaria, cuando se ponen en marcha los "consejos" tipo Freinet; se institucionaliza una hora o un espacio de la vida de la clase en el nivel secundario, con "debates de regulación colectiva e individual".

c. En la enseñanza de las disciplinas: en la enseñanza de la lengua materna se piden "debates de interpretación" orales sobre los textos a partir de diversos episodios; se apunta a desarrollar las capacidades argumentativas a través de discusiones en el marco de algo que por ahora se llama "didáctica de lo oral", que está en proceso de construcción; en educación cívica o educación para la ciudadanía (también llamada "educación para la convivencia") se considera pertinente acudir al debate; en ciencias sociales se debaten las "cuestiones socialmente vivas"; en matemáticas se organizan a partir de "problemas abiertos" los "debates científicos"; en ciencias de la naturaleza o ciencias experimentales se confrontan las hipótesis a partir de enigmas sobre los fenómenos naturales (por ejemplo, el programa de "pequeños científicos"8 que contextualiza en nuestro país las actividades de "La main á la pâte" de la escuela primaria francesa). La educación cívica o educación para la ciudadanía o educación para la democracia y la convivencia (que no es una disciplina, sino una "enseñanza") tiene como uno de sus objetivos explícitos la "metodología del debate argumentado".

\footnotetext{
${ }^{8}$ Ver sitio web que alberga el portal de la Universidad de los Andes: http://pequenoscientificos.uniandes.edu.co/
} 
d. En el dominio oral de la lengua, en el que la discusión es uno de los principales géneros y un indicador fundamental de las dificultades y los fracasos escolares a la hora de atender las exigencias del uso de la lengua que hace la escuela hoy en día.

e. En educación cívica o educación para la convivencia o educación para la urbanidad se juzga prioritario la discusión por razones de la creciente presencia de cierta falta de "urbanidad", de un comportamiento que atenta contra la convivencia; también, porque se pone en juego una ética comunicativa de la persona que aprende en la escuela, y donde la participación democrática de un ciudadano crítico en el espacio público se aprecia valiosa.

f. En la co-construcción de saberes en la clase, que constituye una "comunidad de investigación" o "comunidad de búsqueda" a partir de problemas, enigmas, de cuestionamientos, según el paradigma socio-constructivista de las didácticas disciplinares.

El debate como discusión aparece como su actividad estructurante de manera transversal en la escuela, tanto en el nivel de la vida escolar como en la enseñanza de las disciplinas. Se puede interpretar esta recurrencia y esta insistencia como un síntoma. En el nivel explícito, este éxito podría explicarse porque el debate es a la vez un reencuentro de finalidades juzgadas actualmente decisivas para el sistema educativo.

Ahora bien, en los desarrollos recientes de la enseñanza de la filosofía, la discusión se ocupa sobre lo tratado en el curso magistral o la disertación, pero también se aprecia cómo emerge en el sistema educativo como "discusión de tipo filosófico" ("discussion à visée philosophique") en la escuela primaria y secundaria.

¿Por qué este interés por la forma de discusión de tipo filosófico? Tozzi (2005a y 2007) responde a esta pregunta en términos de: 1) explicación, en el nivel de los hechos; 2) en términos de legitimación, en el nivel de las finalidades de este tipo de prácticas del filosofar, y 3) en términos de un nuevo paradigma en gestación sobre la didáctica del aprendizaje del filosofar.

Respecto al primer punto, el origen y las causas del desarrollo de la discusión de tipo filosófico, se invocan varios elementos:

a. El escenario de fondo es la tendencia de la sociedad a reemplazar paulatinamente una educación que tiene o tenía ciertos tintes autoritarios por un modelo más liberal, tanto en la familia (reducción del autoritarismo) como en la escuela (menos represiva), que le da al infante (alumno) derecho a la palabra, que se preocupa por su opinión a la hora de decidir: la discusión penetra entonces la relación educativa, con un cambio de estatuto de la infancia, como lo testimonia, por ejemplo, la Convención Internacional de los Derechos de la Infancia.

b. Estas evoluciones psicológicas y jurídicas del modo educativo de autoridad han sido epistemológicamente apuntaladas por la innovación en las ciencias de la educación del modelo cognitivista, constructivista y, sobre todo, socio-constructivista del aprendizaje, según el cual la evolución de las representaciones (se dice "opinión" en filosofía), estaría favorecida por la emergencia de conflictos socio-cognitivos en el grupo-clase, lo que llevaría a los programas de cursos o programas curriculares a integrar de manera transversal la discusión como modalidad disciplinar de aprendizaje (ejemplo: el "debate de interpretación" o el "debate científico" en la 
primaria, el debate sobre las "cuestiones vivas" en economía o la "metodología del debate argumentado" en educación cívica en la secundaria).

c. De otra parte, la multiplicación de los comportamientos ciudadanos negativos, la violencia escolar y los problemas de coexistencia de alumnos de orígenes diferentes han llevado a reforzar en el sistema educativo la educación en la civilidad y la ciudadanía, a considerar la discusión en clase (educación cívica) y en la vida escolar (ejemplo: los mediadores) como un método de regulación de conflictos, rehabilitando las formas participativas como el consejo cooperativo de clase, de alumnos, de escuela.

d. El dominio de la lengua materna se presenta como prioridad del sistema educativo, en su doble componente, escrito y (lo que es de nuevo actual) oral, así como la didactización de lo oral, que emerge como un campo de investigación y de prácticas nuevas, en las que el aprendizaje del debate y la discusión es una contribución mayor ${ }^{9}$. La discusión, en tanto forma de expresión oral, es también privilegiada de hecho en la escuela primaria, debido a las dificultades de los niños y jóvenes con la escritura, y porque ella es una actividad colectiva, mientras que lo escrito es individual.

e. Por último, es sobre este fondo de reconfiguración de la sociedad, de una autoridad más participativa en una república democrática, en el que se da una valorización del debate escolar con relación a la construcción de la identidad de un sujeto psicológicamente expresivo (el debate como forma de dominar oralmente la lengua en situación real de intercambio), socialmente cooperativo (el debate como forma regulada de vivir conjuntamente) y cognitivamente confrontado a los otros (el debate como momento de aprendizaje en una actividad de búsqueda). Aquí aparece, sobre un terreno favorable, la discusión filosófica.

Respecto al segundo punto, sin duda, la discusión emerge también en el campo educativo, pero ¿por qué la discusión filosófica? Dos razones se pueden exponer, según Michel Tozzi (2005a), en términos de legitimación, en el nivel de las finalidades o los fines que han facilitado la orientación filosófica de esta práctica:

a. La crisis de las verticalidades tranquilizadoras (trascendencia divina o positivista) y de las utopías alternativas, el relajamiento del vínculo social y el aumento del individualismo han debilitado las personas, horizontalmente condenadas a darse ellas mismas un sentido a su vida, situación que atribuye a la reflexión personal una urgencia colectiva (de ahí la aparición en Francia, desde 1992, de los cafés filosóficos, y el éxito social actual de la filosofía y de la filosofía práctica: publicaciones, universidades populares, etc.). Para el autor francés, esta angustia de época influye sobre la escuela y lleva a que sus niños y jóvenes alumnos, en tanto que pequeños hombres, expresen sus cuestiones existenciales y estén listos para apoderarse de todos los espacios que les permitan abordarlas (lo que va a permitir la discusión).

\footnotetext{
${ }^{9}$ El libro La discussion en éducation et en formation. Un nouveau champ de recherches. Sous la direction de Michel Tozzi et Richard Etienne. Paris: L'Harmattan, 192 páginas, busca cuestionar y preguntarse por el recurso a la discusión que se desarrolla en diversos espacios de la educación y de la formación. ¿Se puede utilizar la discusión para educar y formar? ¿Favorece ella los movimientos del pensamiento, la construcción de conocimientos, la adquisición de conocimientos y cuál pertenencia puede tener la discusión para producir o generar un compromiso ciudadano?
} 
b. Un elemento exterior también favorece su introducción: la preexistencia del método Lipman (lectura, selección de una cuestión, discusión, etc.), con sus novelas y sus libros del maestro, desarrollado desde 1970 en los Estados Unidos e introducido en Québec en 1982, en Bélgica en 1985, en Colombia a través de la actividad del profesor Diego Antonio Pineda, y en general en América Latina desde 1999 (Kohan y Wakman, 2000). Método valorizado por el departamento de "filosofía y ciencias humanas" de la Unesco (2007:1-45) y que crea el precedente de una "filosofía para niños", que iría a permitir autorizar esta práctica, pese a la oposición de la institución filosófica y de la tradición antigua de su enseñanza.

Finalmente, respecto al tercer punto: un nиevo paradigma en gestación. Hablar de esta gestación en la enseñanza de la filosofía, según Tozzi (2007:211-215), implicaría apoyarse de hecho sobre las nuevas prácticas filosóficas ${ }^{10}$ que han surgido a finales del siglo XX en Francia y otros contextos ${ }^{11}$. Sin embargo, agrega Tozzi, esta "desespecialización" de la filosofía en la escuela y en la ciudad, su "desescolarización" en las aulas a través de esta forma innovadora constituye para los guardianes del templo filosófico más bien un abuso del lenguaje y una traición a las exigencias de la disciplina. Para este grupo no se trata de filosofía, sino más bien se trata de desarrollar el pensamiento reflexivo, o de conocimiento (oral) de la lengua -con el consiguiente peligro de desembocar en la retórica sofistica-, o de educación para la ciudadanía, o de construcción psicológica de los individuos, objetivos por otra parte encomiables, pero que pueden instrumentalizar la filosofía como disciplina (2007: 213).

Ahora bien, estas nuevas prácticas filosóficas tienen el interés de estimular la reflexión sobre diversas cuestiones difíciles: a) el papel de la filosofía en la polis democrática, renovando el contacto socrático sobre el ágora (el espacio público) ${ }^{12}$; b) el momento y el papel de la filosofía

\footnotetext{
${ }^{10}$ Las "Nouvelles Pratiques Philosophiques" (NPF) es el término que se utiliza con más frecuencia en los países francófonos para referirse a ese conjunto de prácticas filosóficas de reciente creación (como el movimiento de los cafés filosóficos, de los talleres de filosofía o de las universidades populares) que han renovado el modo de "hacer filosofía" dentro y fuera del aula. Para ampliar la información sobre estas prácticas, puede consultarse en español el artículo de Gabriel Arnaiz, publicado en el diccionario de didáctica de la filosofía de Enzo Ruffaldi (voz "Nouvelles Pratiques Philosophiques. En: http://www.filosofiamo.com/), o la introducción, del mismo autor español, al libro de Oscar Brenifier El diálogo en clase (Idea, 2005) titulada "Nuevas Prácticas filosóficas: de los cafes filosóficos a los talleres de filosofía"; disponibles en Internet: http://www.redeseducacion.net/Art_GabrielArnaiz.htm. También puede verse del mismo autor: "Evolución de los talleres filosóficos: de la filosofía para niños a las nuevas prácticas filosóficas" en: http://www.filoeduc.org/childphilo/n5/GabrielArnaiz.htm.

${ }^{11}$ Tozzi argumenta como: (a) desde 1992, el movimiento de los "cafés filosóficos" se ha tomado al píe de la letra esa fórmula de Diderot que exclamaba: "!Popularicemos la filosofía!" y la está aplicando para la polis y en la polis. Con un público heterogéneo que acude voluntariamente, los cafés filosóficos han inventado una nueva fórmula de filosofía en el ágora, con prácticas muy diversas, con exigencias intelectuales desiguales según los animadores y los públicos, consistente básicamente en desarrollar los intercambios de un grupo de personas a partir de unas cuestiones de interés antropológico o social, y funcionando como una especie de intelectual colectivo que se debe enfrentar a un problema difícil. (b) Los talleres de filosofía que se inician primero en centros de primaria en 1996 de la mano de J. Lévine y A. Pautard, y después en secundaria (especialmente con alumnos con dificultades, han desarrollado al margen del programa oficial una serie de prácticas innovadoras que reivindican su cariz filosófico. Estas prácticas, basadas en la discusión y reflexión oral, e inspiradas a partir de 1998 por el método de M. Lipman, actualmente adoptan la forma de una articulación entre debates de interpretación en francés sobre obras de literatura juvenil y de discusiones de tipo filosófico, según la formula admitida desde hace tiempo.

${ }^{12}$ En el artículo "café-philo et prof de philo" (L'Agora, $\left.n^{\circ} 12,2001\right)$, Michel Tozzi afirma que "el filósofo de profesión o el profesor de filosofía tiene la responsabilidad de ejercer, fuera de la escuela y en la polis, un papel al mismo tiempo filosófico y cívico", siendo el café filosófico una de las posibles vías del filósofo para desarrollar su
} 
en la educación: ¿existe una "educabilidad filosófica de la infancia o de la adolescencia”, una edad para filosofar?; c) la definición del tipo de competencias constitutivas del pensamiento reflexivo: ¿en qué consiste eso de "filosofar", qué proceso de pensamiento requiere y cuáles son sus exigencias intelectuales?, y d) ¿la determinación de los procesos de aprendizaje de la actividad filosófica y de la didactización de la disciplina pueden favorecer este aprendizaje?, ¿puede filosofarse discutiendo? y, en caso afirmativo, ¿cuáles son las condiciones para ello y cuál es el papel del profesor?

\section{Aproximación filosófica y didáctica de la discusión con perspectiva filosófica}

La discusión y en particular la discusión filosófica remiten en nuestros días a diversas prácticas y escenarios educativos formales e informales. Podría ser útil para la investigación didáctica analizar estas prácticas, entre otras razones, para determinar si estas discusiones son o no filosóficas: si no lo son, por qué, y si lo son, en qué ${ }^{13}$.

La "discusión filosófica" aparece entonces como una expresión por definir (tarea filosófica: conceptualizar la noción) y, desde un punto de vista problemático, como un conjunto de cuestiones que se deben trabajar. Por ejemplo: a) ¿es un concepto nuevo, solidario de la modernidad (con relación a y por oposición con el diálogo de la Antigüedad o con la disputatio de la Edad Media); b) una discusión filosófica en varios aspectos, con relación al diálogo, ¿hay condiciones de posibilidad?, si las hay, ¿cuáles son las de una discusión fílosófica?, ¿cuáles son los presupuestos?; c) ¿es deseable practicar las discusiones filosóficas en clase, y en la vida pública (enfoque praxeológico)? Puede ser ella un "género filosófico", en el sentido de E. Ruffaldi $?^{14}$. ¿El ideal regulador, en el sentido kantiano, de una forma de pensamiento del cual deberían inspirarse las prácticas filosóficas y pedagógicas? Y si es así ¿cómo (proceso praxeológico de didactización)? ¿Cuál sería, por ejemplo, el estatus de la discusión filosófica con

deber cívico. El propio Tozzi anima desde hace diez años un café filosófico en Narbonne, así como un taller filosófico para adultos desde el año 2004 en la recientemente fundada Universidad Popular de Septiemanie (http://www.perso.orange.fr/universitepopu.septi/), siguiendo el ejemplo de la famosa Universidad Popular de Caen (http:// perso.orange.fr/michel.onfray/accueilup.htm) fundada en el 2002 por el polémico Michel Onfray. Desde entonces y hasta ahora, son seis las universidades populares que se han creado en Francia y Bélgica. Para más información, puede leerse el artículo de M. Tozzi "L’atelier de philosophie pour adultes á l'université populaire de Narbonne" (L'Agora, $\left.n .^{\circ} 28,20069\right)$.

${ }^{13}$ Para el desarrollo del apartado 3, tomo los argumentos expuestos por Michel Tozzi (2005a) en: Place et valeur de la discussion dans les nouvelles pratiques à visée philosophique. Recuperado el 30 de julio de 2008, en http.//www.philotozii.com/ ?p=249.

${ }^{14}$ Ruffaldi define el "género" como: "Analogamente a quanto avviene in letteratura, per "generi filosofici" si intendono le diverse modalità di scrittura considerate dal punto di vista della forma. L'espressione che abbiamo scelto per questa voce è forse meno immediatamente comprensibile di quella, in sé più chiara ma meno corretta, di "generi letterari in filosofia". È indubbio che in filosofia esistono molti generi diversi (dal dialogo alla confessione, dall'epistola al trattato, ecc.) anche se si è dedicata minore attenzione a questo fatto rispetto a quanto avviene in letteratura. Ciò sembra riconducibile, fondamentalmente, a due ordini di motivi: da un lato all'uso meno sistematico dei testi nella didattica della filosofia, dall'altro lato al carattere fondamentalmente argomentativo dei testi filosofici che sembra costituirne un tratto unitario prevalente sulle differenze di genere.>> (ver: voz: "Generi Filosofici" Dizionario di didattica della filosofia http://www.filosofiamo.com/dizionario_zoom.aspx?pubblicazione.id=1006\&) 
relación al curso del profesor, al texto de un autor, a la disertación ${ }^{15}$. ¿Cómo articular la discusión con otras modalidades de enseñaza-aprendizaje del filosofar?

\subsection{Qué no es la discusión filosófica}

Parecería más fácil entre colegas de filosofía ponerse de acuerdo sobre lo que no es la discusión filosófica:

a. Un simple intercambio de opiniones en el que cada uno dice espontáneamente lo que piensa, es decir, exposición de prejuicios; una conversación sin ton ni son, sin orden ni concierto, derivada en últimas del pensamiento asociativo a propósito de intereses privados o de temas públicos del momento, sin otra finalidad que el placer de hablar, esto es, de deslumbrar en compañía.

b. Un intercambio erístico (en el sentido aristotélico), conflicto de ideas sin escucha mutua, enfrentamiento entre personas o confrontación con base en la manipulación sofística, que busca con(vencer) al otro o a los espectadores por cualquier medio eficaz (persuasión, sofisma, argumento ad hóminem), del tipo debate electoral entre candidatos.

c. Un debate mediático de periodista sobre un "tema de sociedad", en el que el animador yuxtapone los puntos de vista cortados en tiempos muy limitados, sin el tiempo de la reflexión ni de la exposición de un pensamiento, sin interactividad real entre los participantes.

d. Un debate judicial, que en un proceso asegura el derecho de expresión de la defensa y de la acusación, la convocatoria de testigos y de expertos, cualifica los actos con referencia a la ley y termina sobre una decisión de jurisprudencia en lenguaje preformativo: el veredicto.

e. Una discusión psicológica, del tipo psicoterapéutico verbal de grupo; la palabra psicológica se toma bajo la forma del relato personal, expresa la singularidad de una historia individual a través de sus efectos, es comunicación del consciente al inconsciente, se hace ecolalia o se calla, y la escucha psicológica, por su empatía existencial, entiende el sufrimiento de una persona, mientras que la palabra filosófica se origina en la razón y se dirige a ella, trabaja el concepto, y la escucha filosófica es ante todo cognitiva y crítica.

f. Un debate democrático, que abre un espacio público de discusión en la ciudad sobre los problemas políticos (lo que es filosóficamente interesante, pero restrictivo en cuanto al campo); aclara, por el pluralismo de las opiniones (y queda en un nivel doxológico) y confronta el punto de vista de los ciudadanos, o el de sus representantes, entre ellos o con el punto de vista de expertos; puede convertirse, por los desafíos de poder, en demagógico

\footnotetext{
${ }^{15}$ Para una presentación de la disertación en la enseñanza y didáctica de la filosofía, cfr.: Dreyfus, Dina; Raffin, François (1994) La dissertation philosophique. La didactique à l'œeuvre. París: INRP-CNDP-Hachette. 143 páginas. Gómez Mendoza, Miguel Ángel (2003): "La disertación en la enseñanza de la filosofía". Introducción a la didáctica de la filosofía. Pereira: Editorial Papiro-Universidad Tecnológica de Pereira, pp. 39-56. Gómez Mendoza, Miguel Ángel (2005): Didáctica de la disertación en la enseñanza de la filosofía. Métodos y procedimientos. Bogotá: Cooperativa Editorial Magisterio. Colección Didácticas Magisterio. 100 páginas.
} 
(interesarse en el número más que en la verdad); se trata a menudo de discutir para decidir a nivel individual o colectivo (ejemplo: votar); actuar y no solamente pensar; se aprecia la mayoría, y no lo universal; el pensamiento democrático es el de un grupo, de un Estado; el pensamiento filosófico es singular, pero tiene alcance universal, por racional, mientras que la multitud es con frecuencia pasional.

g. Un debate científico: hay aquí, como en filosofía, una ruptura con la opinión (Bachelard), el sentido del cuestionamiento, la emergencia de los problemas, el tratamiento racional de las preguntas, la voluntad de las respuestas fundadas, una discusión pública entre pares (y no en el ágora), donde toda afirmación es examinada como hipótesis por confirmar o invalidar; pero las preguntas se plantean en el campo del conocimiento (excluyendo la ética, la estética y la metafísica); el lenguaje incluye codificaciones formales (ejemplo: fórmulas matemáticas, mientras que toda discusión filosófica privilegia y casi siempre se lleva exclusivamente en lengua natural); el modo de administración de la prueba recurre a la demostración (con su modo deductivo indiferente si lo toma en cuenta el auditorio), a la observación (construida) o a la experimentación, al contrario de la argumentación filosófica racional, pero no científica; la posición científica del problema presupone en derecho la posibilidad de una respuesta, mientras que la cuestión filosófica no se satura, es susceptible de muchas respuestas, sin que haya, por tanto, relativismo, porque permanece la interpretación de un horizonte de universalidad y de verdad); la pregunta científica y su respuesta llama a una respuesta "consensual" en la comunidad de expertos, al menos provisionalmente; mientras que las "soluciones" en filosofía son siempre plurales, por ejemplo, la filosofía de Platón, no sería obsoleta.

\subsection{Algunas condiciones de posibilidad de la discusión filosófica}

Intentemos ahora, siguiendo a Tozzi (2005a), destacar algunos criterios, atributos, presupuestos, condiciones de posibilidad de una discusión específicamente filosófica. Tarea compleja que supondría definir qué es una discusión (por ejemplo con relación al diálogo), lo qué es la filosofía (consenso imposible de encontrar entre los mismos filósofos), lo que es una discusión filosófica (se ha visto lo que ella no es), qué es lo que en ella es específicamente filosófico. El desafío didáctico es importante: ¿cómo practicar discusiones filosóficas en clase o en otros escenarios, si no se tiene claridad sobre lo que es o debería ser una discusión filosófica? ¿Cómo, de otra parte, cualificar una discusión en clase de filosofía? Veamos a continuación algunos elementos de posibilidad de la discusión filosófica:

a. Un "discutidor" filosófico mantiene una cierta relación con la verdad. Es la investigación de una verdad la que da filosóficamente sentido. Los "momentos filosóficos" de una discusión son aquellos en los que esta finalidad aparece claramente: cuando un individuo, los interlocutores o el grupo se somete voluntariamente, individualmente o colectivamente, a una exigencia de verdad. Cuando el desafío es especulativo, relacionalmente desinteresado: no se trata entonces de manipular al otro, sino de tener respuesta a su propia cuestión, es la presencia del deseo de saber y en consecuencia de no saber, asunción de la deuda, atrevimiento por la vulnerabilidad cognitiva. Cuando se llega intelectualmente convencido a la discusión filosófica, se le da a esa afirmación estatus de simple hipótesis por examinar, escuchar, criticar. Discutir filosóficamente es tener el sentido de la fragilidad de una convicción, la fuerza de confrontar, el riesgo y la esperanza de que ella sea discutida, puesta en entredicho, para ir más lejos. Es admitir que su 
"opinión" es a priori y en derecho discutible, que no se puede ser racionalmente portador de ningún dogma. No hay discusión sin el postulado del ser discutible (discutabilité) de toda afirmación. Lo que plantea el problema de la creencia en una discusión. La condición de no ser discutible no es filosófica, sino religiosa (el dogma de la revelación implica una fe), o científica (por ejemplo una demostración matemática en el marco de un axioma dado).

b. Esta relación con la verdad, al menos como horizonte, entraña una cierta relación con lo real. Si se le discute es para aclarar una cuestión que se esboza sobre lo que se plantea por lo real, concretamente sobre mi relación con el mundo, del otro conmigo, con Dios. No se discute filosóficamente ni por nada ni sobre nada. Hay un desafío: la relación de juicio a lo real y a la verdad. La posibilidad para el pensamiento de decir la verdad, de pensar lo real.

c. De ahí una relación esencial y necesaria, en toda discusión filosófica, en el tema abordado, en el objeto-sujeto de la discusión, en las cuestiones planteadas sobre este objeto de pensamiento y en el tipo de pregunta. Toda cuestión no lleva una discusión filosófica. Por ejemplo una cuestión factual sobre los conocimientos declarativos (ejemplo: el dato de la Batalla de Boyacá remite a una respuesta única e indiscutible). La pregunta planteada en una discusión filosófica debe tener sentido para todos y cada uno, comprometer los desafíos antropológicos, ser planteada o entendida en uno de los campos de reflexión filosóficos (ejemplo: epistemología, ética, política, estética, metafísica...), no puede ser resuelta científica o técnicamente, o jurídicamente. Ella debe ser también controvertida, susceptible de varias respuestas fundamentadas. Es entonces una cuestión abierta, que atraviesa los siglos con reformulaciones y desplazamientos, ella no es clausular, en el sentido de cerrar o terminar un periodo, en este caso, poner fin a lo que se estaba diciendo. De hecho, como la condición de posibilidad de una objeción hace todo enunciado filosófico en derecho contestable (controvertible), una discusión filosófica es no solamente inacabada, sino potencialmente inacabable. Ella se detiene porque es la hora de detenerse, pero podría continuar. Otros continuarán abordándola y respondiéndola.

d. La agudeza de este cuestionamiento implica cierta relación con la libertad, que compromete la relación del sujeto con lo que él dice, una relación consigo, con el lenguaje y su pensamiento; lo que pone en juego, más allá de los enunciados proferidos, su modo de enunciación; discutiéndolo adopta una cierta postura intelectual y moral; autenticidad de una actividad de búsqueda y de apertura; actitud de cuestionamiento; exigencia y humildad a la vez; sentido de la complejidad; habitar una cuestión y ser habitado por ella; sentirse requerido. De ahí, si hay en una discusión filosófica una relación con el dominio por la voluntad metódica de saber y una palabra reglada y regulada, hay también una relación de desprendimiento, un desbordamiento por la profundidad de la cuestión, la perplejidad frente a las soluciones, el sorprenderse por las objeciones, lo aleatorio de la interacción; hay compromiso interior más allá de la exterioridad y del formalismo; ejercicio de juicio y emancipación por el uso de la razón.

e. Porque la relación con la razón caracteriza una discusión filosófica. Esto es, una actitud razonable que trabaja sobre sí misma para dominar la emoción que podría inundar y turbar el juicio. Esta relación implica ponerse en posición del yo filosófico que existencialmente implica un locutor universal; si bien todo pensamiento es singular, personal y contingente, se dirige a un "auditorio universal", con la pretensión de validez de lo dicho y la legitimidad discursiva de la intervención. La relación con la razón implica ponerse en el lugar de "todo otro" y buscar el "mejor argumento". Esta exigencia de racionalidad se actualiza por los procesos intelectuales de 
conceptualización de nociones (saber de lo que se habla, definir, operar las distinciones conceptuales), de problematización (cuestionar las evidencias, derivar las posturas, interrogar los presupuestos y consecuencias), de argumentación (fundar, reconstruir, objetar, saber si lo que es dicho es verdadero) ${ }^{16}$.

f. En una discusión filosófica, la relación con el pensamiento es relación con la razón, porque esta relación se expresa a través del lenguaje oral, en la confrontación con el otro y con un grupo, lo que le confiere su especificidad.

g. Esta relación con el lenguaje es constitutiva del pensamiento, porque en principio, se puede pensar con y sobre las palabras. Saber de qué habla para poder pensar. Cuando hablar quiere verdaderamente decir alguna cosa, ya que se trata de la relación del lenguaje con la verdad. Pensar lo que se dice, y no contentarse con decir lo que se piensa; pensar en lenguaje natural, y no de manera formalizada, científicamente, codificado, pese al ideal de demostración del positivismo lógico, pensar en el lenguaje oral.

La expresión oral de un pensamiento difiere de su expresión escrita. Lo oral, en el surgimiento de la palabra encarnada, lineal, invita a la improvisación, para lo mejor y para lo peor. No obstante, la relación con el pensamiento se hace en una discusión filosófica en interacción social. Esta es la que descentra, sorprende, desmonta cantidad de respuestas, y luego inventa. Se debe estudiar esta relación entre pensamiento, palabra e interacción. No se piensa hablando, como escribiendo; no se piensa solo como se piensa en interacción verbal con el otro. Comparemos el pensamiento solitario frente a la hoja en blanco en el escritorio u oficina, y la discusión filosófica. ¿Cómo se construye el pensamiento en una discusión filosófica, en esta dialéctica entre el encararse consigo mismo y con los otros, en la relación con su propia palabra convocada por la dinámica de los intercambios? ¿La sucesión de los movimientos de introversión y extroversión, el dialogismo de las intervenciones, la fuerte intertextualidad de lo que se dice muestran una construcción original, no lineal, todo un retomar de respuestas, de concesiones, de matices? En el fondo, ¿cómo se piensa de manera conjunta separadamente en una discusión filosófica? Se podría sostener que la discusión filosófica es un peligro para el pensamiento; mucha ligereza de los interlocutores (a menudo "doxicos") con relación a la profundidad de los autores; mucha dispersión con relación a la multiplicidad de los locutores, en el lugar y espacio de una lógica expositiva estructurada; mucho de imprecisión en el surgimiento espontáneo de lo oral con relación a la seriedad y precisión de lo escrito; mucha velocidad en los intercambios con relación a la "paciencia del concepto". Pero alguna cosa se juega aquí en la relación con el pensamiento, desde que la discusión filosófica es una "comunidad de búsqueda" (Lipman), cuyas interacciones sobre una cuestión alimentan la reflexión de cada uno y el trabajo colectivo.

16 Una exposición detalladas, acompañada de un ejercicio para determinar la presencia de estos procesos intelectuales se encuentra en el capítulo 7 (<<Apprendre à philosopher. C'est quoi? Et comment?: « Articuler trois processus de pensée, Connaître et mettre en œuvre ces processus :-Problématiser, Conceptualiser, Argumenter-, Repérer ces processus de pensée dans un texte philosophique. »>>) del libro de Michel Tozzi: (2005) Penser par soimême. Initiation à la philosophie. Lyon: Chronique Sociale, pp. 143-175. 
Se dirá que los alumnos gustan del discutir porque tienen la impresión de no "trabajar", evitando en este momento confrontarse con un curso, un texto o la escritura, o porque es la ocasión de una afirmación adolescente de sí mismos. Pero no se debería subestimar el interés de la confrontación de ideas, que amplía su horizonte.

h. La discusión filosófica compromete en efecto una relación con el otro. Es el caso de todo pensamiento: Descartes llama sin cesar las objeciones del lector, y Kant solicita su juicio. Pero aquí se trata del otro de carne y hueso, no de un lector ("lectorat") universal y potencial como en el ensayo o un destinatario preciso como en la carta filosófica. El otro, o más exactamente varias personas, con las cuales cada uno entra o puede entrar en interacción social verbal (como, por lo demás, no verbal y paraverbal), en una comunicación presencial y cercana, al contrario de la mesa redonda moderna, donde los soliloquios se yuxtaponen, que invita a improvisar sobre el campo una respuesta coherente.

La discusión filosófica está presente aquí, porque se debe co-construir las relaciones de sentido, ruptura de fuerzas entre los individuos: relación con la fuerza física a través del lenguaje; pero también relación de fuerza verbal (injuria, tentativa de manipulación sofística), cuando se ve en el otro un colaborador, y no un adversario. Hay entonces respeto de la persona y de sus ideas, tolerancia a la diferencia, aceptación del derecho de expresión del otro, y del pluralismo de los puntos de vista.

Pero hay más: necesidad del otro para buscar, interés por su visión del mundo, tener en cuenta sus opiniones, llamado a su juicio, a su crítica, por su pertenencia a la comunidad de los espíritus racionales, que abre un espacio público de confrontación universal. La discusión filosófica supone la posibilidad de ser "alterado" (modificado) por el otro. Hay entonces en la discusión filosófica un doble "cuidado" del otro.

i. Lo que compromete una ética comunicacional. Desde el punto de vista de la relación con la verdad, se trata de una moral del pensamiento, ya que cada uno debe libremente someterse a las constricciones de la razón, a las exigencias de la lógica y de la coherencia, a la fuerza de las objeciones. Se trata de dar razón de su posición, y de dar razón al "mejor argumento". Desde el punto de vista del otro, se trata de respetar su palabra y su persona, pero también de tener en cuenta "la parte de verdad" que él abre en mí. Se trata, en fin, es esto la estima intelectual, de plantearle las cuestiones y de dirigirle las objeciones más aptas para hacerlo progresar. Desde el punto de vista del sujeto tratado, cada uno tiene, en una discusión filosófica, la responsabilidad de llevar intelectualmente el problema planteado, de hacer avanzar colectivamente su tratamiento con rigor, de callarse también cuando no hay nada decisivo que decir. Esta responsabilidad es asumida frente al grupo, donde se debe también respetar las reglas de funcionamiento para que la discusión filosófica sea posible.

j. Porque hay en una discusión filosófica, en la medida en que la interacción verbo-conceptual es plural, una relación con el grupo de discusión. ¿Se puede discutir filosóficamente con treinta o cincuenta personas? ¿Es que la palabra compartida (no la palabra ex cátedra, que soporta muy bien el gran auditorio de clase universitario) cambia de naturaleza o de estatus con el número o cantidad? ¿Cómo regular la palabra filosófica en un grupo numeroso, cuando la informalidad 
sería cacofónica? Es este problema del número el que plantea la cuestión de la relación entre debate democrático y discusión filosófica ${ }^{17}$.

\section{Cierre: desafíos, tentaciones y derivas de la discusión filosófica en el aprendizaje del filosofar}

La discusión filosófica, si se comprende desde un punto de vista grupal, esto es, desde una comunidad de búsqueda, que apuesta al desafío de poder profundizar colectivamente un problema, tendría que enfrentar ciertas tentaciones y desviaciones, que Tozzi (2005a) describe con los siguientes elementos:

a. En el nivel intelectual, la superficialidad (todo depende de la calidad de las intervenciones y de la exigencia reflexiva de los participantes y del animador/profesor), y la dispersión (temas fuera del punto, incoherencia de las intervenciones mutuas, multiplicación de las preguntas, de los contenidos y las nociones), lo que trabaría el rigor del análisis y el avance de una progresión de la discusión.

b. En el nivel psico-sociológico, porque la discusión filosófica pone en juego la vida de un grupo, y se puede generar un apuntalamiento narciso de un yo "más psicológico que filosófico", erudición cultural que se da por conocida, esto es, el modo alusivo (ejemplo: "como lo saben todos ustedes, Wittgenstein, en el Tractatus Logico-philosophicus"), o el surgimiento de líderes que se toman la palabra o de conflictos socio-afectivos parásitos con relación al trabajo del pensamiento, particularmente emergentes o presentes en los adolescentes.

Es una cuestión abierta saber si una discusión filosófica es producto o puede producir un "pensamiento colectivo", a través de sus intentos de conceptualización de nociones, de elaboración de problemáticas, de preguntas, de tesis, de argumentos, de acuerdos y desacuerdos. Con seguridad no un pensamiento colectivo, en el sentido de que la discusión (en clase o en el café) produciría conceptos filosóficos, filosofía y doctrina. Como muy bien lo sintetiza Tozzi (2005a), "se lleva a cabo en clase un aprendizaje; donde se piensa que no se trata de filosofía, porque se está precisamente en proceso de aprender, o donde se piensa que se trata ya de

\footnotetext{
${ }^{17}$ Por razones del alcance y los objetivos de esta comunicación, se pueden sugerir aquí dos asuntos o cuestiones que se debaten actualmente en la perspectiva de la discusión con fines filosóficos: (a) La discusión filosófica y el debate democrático. Se pregunta Tozzi (2005a): “¿Si el pueblo piensa según la opinión, y la filosofía pretende superar la doxa, entonces la filosofía y, en consecuencia, la discusión filosófica no tienen ellas una naturaleza intelectualmente aristocrática? Platón concluía que la ciudad debería ser gobernada no por el pueblo, sino por los sabios. La ilustración y los pensadores de la república desplazaron la problemática, a través de la instauración de una escuela para todos, donde la filosofía identificada con la causa de la escuela del pueblo debe ser enseñada a cada uno. El alumno no es más un discípulo que escoge su maestro, sino un futuro ciudadano obligado a ir a la escuela y a hacer filosofía para instruirse y ser aclarado. Se debe entonces hacer la filosofía accesible al pueblo (instaurar una "demosofía"), especialmente para el aprendizaje de la discusión filosófica". (b) Discusión filosófica y comunidad de búsqueda. "El interés de una discusión filosófica es intentar instaurar un grupo de personas en 'comunidad de investigación' sobre un problema esencial. Lipman emplea esta expresión con relación a la filosofía para niños para la cual propone un procedimiento en los años 1970. Se trata para el docente de constituir su clase en "comunidad de investigación", es decir, en un lugar y en un momento donde cada participante intenta aportar su grano de arena a la problemática planteada, ubicándose en un punto de vista racional, teniendo en cuenta su edad, su madurez y sus conocimientos" (Tozzi, 2005a).
} 
filosofía, porque se comienza precisamente a aprender. Se debe evitar el malentendido: una disertación puede esbozar una actividad reflexiva sin que por lo tanto presente o muestre doctrina o sistema".

No obstante, se podría afirmar que lo que puede haber en una discusión filosófica es sólo una yuxtaposición, o al menos una confrontación de puntos individuales. Acaso, ¿todo pensamiento no es singular, personal? ¿No hay solamente trabajo de cada uno en común, antes que trabajo en común? No se está acaso en la situación de "pensar en conjunto pero de manera separada separadamente". En todo caso, al margen de estos cuestionamientos, el trabajo de la discusión en grupo sería una feliz ocasión para preguntarse, de trabajar intelectualmente, ya sea por escucha activa o por interacciones efectivas. Este es el punto de vista del participante, que construye su propio pensamiento a menudo a partir de lo que escucha, recibe, intercambia.

No obstante, hay más cuestionamientos: ¿la participación ideal acaso no es la de aquel que busca hacer avanzar la reflexión sobre el problema que concierne a cada uno de los participantes, a todo el grupo, más allá de la comunidad de mentes, porque en esa participación se avizora la racionalidad universal? La participación ideal ¿no es la de aquel que intenta salir de "su" punto de vista para hacer acceder al grupo a la claridad cognitiva y al mejor argumento, aquel que cree en una posibilidad en derecho al acuerdo racional?

Ahora bien, si se acepta que el rol de un animador de la discusión filosófica, como comunidad de búsqueda, es el de construir sentido, relacionando y poniendo en perspectiva las intervenciones mutuas de los participantes; sin dejar de lado la relación con el tema, esto es: de nombrar lo que se hace (tentativa de definición, emergencia de una tesis, producción de argumento, ilustración por un ejemplo, objeción por el contrario-ejemplo, aparición de otra tesis, desplazamiento de la pregunta, apertura de un nuevo campo de reflexión, etc.); de volver a centrar sobre el problema, de volver a lanzar los cuestionamientos, de pedir una profundización, y de hacer perceptible este sentido a los participantes, se dirá entonces que el sentido es construido por el animador/profesor antes que por el grupo o sus participantes.

Puede ser cierto. Pero una reformulación del profesor o animador parte de una intervención real, de una síntesis parcial o final, oral o escrita a posteriori, que vuelve a tomar lo que ha sido efectivamente dicho, y capitaliza un avance colectivo. Es difícil separar o dividir la eventual yuxtaposición de intervenciones individuales y seleccionar entre ellas en función de la elaboración de su propio pensamiento, y una co-elaboración colectiva, significativa de la comunidad de investigación o búsqueda como "intelectual colectivo". Toda síntesis es la de un individuo que estructura y reelabora, y por lo tanto intenta dar cuenta de un trabajo común. En esta perspectiva se trata de retomar con la máxima objetividad posible todo lo que ha sido dicho de significativo, teniendo el mismo animador cierto dominio del tema abordado. Se hablará en este caso de co-construcción de sentido: alguna cosa surge del trabajo en grupo, que no es el pensamiento del profesor; si bien el pensamiento sigue siendo personal, no obstante puede superar lo que piensa cada uno de los participantes, por la riqueza de los intercambios.

Se puede, evidentemente, preguntar si no hay actitud demagógica de un profesor cuando reúne en una síntesis las opiniones de los alumnos, descuidando su tarea de "elevar" las opiniones de los alumnos. Una discusión filosófica puede dar un enfoque o perspectiva de pensamiento gracias a la confrontación. La discusión filosófica no es y no puede ser el todo de la enseñanza filosófica. 
El profesor tiene que confrontar allí donde cada uno y el grupo está, confrontar su propio curso y los textos de autores que se emplean.

En este contexto se comprenden mejor ahora los obstáculos de la discusión filosófica (Tozzi, 2005a), y esto en dos sentidos: a) no asumir las exigencias intelectuales de un pensamiento: no elaborar un cuestionamiento, no estar habilitado por la pregunta, responder a una pregunta sin preguntar por sus presupuestos y consecuencias, emplear las palabras sin definirlas, tomar un mal ejemplo para un argumento, caer en la anécdota, contar y contarse, afirmar sin fundamentar, pegarse a su idea, contradecirse, no saber objetar o responder a una objeción, rechazar, someterse libremente a la razón, no expresar en tanto que interlocutor ni dirigirse a un auditorio universal, no sentirse integrante de una comunidad de espíritu; b) no asumir las exigencias de una discusión intelectualmente clara en sus fines: no llegar a comprender al otro (porque nos molesta o nos hace salir de nuestro marco de pensamiento), ubicarse en el punto de vista del otro (y de cualquier otro), no tomar en cuenta sus objeciones, no estar abierto a las pistas nuevas que él propone, hablar para ubicar su palabra y no para hacer progresar el debate, dar las referencias eruditas sin explicitarlas, dar las lecciones a los otros, querer "salvar las apariencias", no aceptar las reglas democráticas de un grupo de discusión (ejemplo: intervenir salvajemente cuando no se tiene la palabra, cortar la palabra a alguien, hablar largo tiempo, juzgar y atacar las personas en lugar de criticar las ideas, ser intolerante, etc.). Lo que remite a la responsabilidad de cada participante en la discusión filosófica, y en particular al animador o profesor, para hacer respetar estas exigencias.

Actualmente, afirma Tozzi,

...nos encontramos con la siguiente situación: la coexistencia y la confrontación entre un modelo tradicional de enseñanza de la filosofía en la escuela secundaria, e incluso en la universidad, la primera seriamente criticada, y una serie de prácticas sociales y escolares emergentes, no institucionalizadas, pero portadoras de una gran normatividad creadora que puede ser esclarecida por una ética comunicativa de inspiración habermasiana, encontrando un eco favorable en numerosos alumnos, maestros y formadores. El diálogo es a veces difícil, y las posiciones contradictorias: para algunos, se trata de un intento de subversión de la disciplina; en el mejor de los casos por desconocedores de buena fe de las exigencias propias de la filosofía, en el peor de los casos por "filo-traidores" de la pedagogía; los alumnos no están suficientemente maduros, ni poseen los conocimientos, lo que único que estaremos haciendo será convertir la clase en una tertulia de café. Sin embargo, este diálogo entre dos partes puede ser constructivo: por un lado, los filósofos curiosos, sin prejuicios a priori, que cuestionan la doxa corporativa de la tradición francesa (muy diferente de la de otros países), incluso los fundamentos de su propia identidad profesional, y por el otro, los partidarios de estas nuevas prácticas filosóficas, que raramente poseen una sólida formación filosófica, conscientes de las exigencias que requiere el pensamiento para incrementar la filosoficidad de su práctica. A este tipo de diálogo heurístico es al que nosotros, por nuestra parte, intentamos contribuir, puesto que el compromiso común de todos no es otro que la formación de ciudadanos reflexivos y críticos (2007: 214-215).

Desde una perspectiva didáctica general, más allá de la "discusión de tipo filosófico", se concluye con Tozzi (2004: 11-12) que el debate, como expresión de la "discusión", se didactiza en la escuela como objetivo de aprendizaje ("aprender a debatir"), por ejemplo en la didáctica 
oral de la lengua materna, para dominar un género de lo oral como "género escolar"; en educación cívica, para saber intervenir en el "espacio público escolar" de la clase y la institución educativa. Pero también como medio de aprendizaje disciplinar de saberes y de saber-hacer, entre ellos los filosóficos, y de socialización democrática para "vivir juntos o en convivencia aprendiendo".

\section{Bibliografía}

ARNAIZ, Gabriel (2007): “QQué es la filosofía práctica?”. AParteRei. Revista de filosofía. [En línea]. Recuperado el 14 de agosto de 2008, en http://serval.pntic.mec.es/AParteRei

Cifuentes, Luis María (1997): "Materiales de filosofía". En: Luis M. Cifuentes; J. M. Gutiérrez (Coordinadores): Enseñar y aprender filosofía en la educación secundaria.. Barcelona: ICE-Horsori editorial. Pp. 137-156.

DoRTU, Véronique (2004): «Histoire belge des cours dits philosophiques». Diotime l'Agora n. ${ }^{\circ}$ 21, avril.

DREYFUS, Dina; Raffin, François (1994): La dissertation philosophique. La didactique à l'ouuve. Paris: INRP-CNDP-Hachette. 143 p.

Gómez MendozA, Miguel Ángel (2003): "La disertación en la enseñanza de la filosofía". En: Introducción a la didáctica de la filosofía. Pereira: Editorial Papiro-Universidad Tecnológica de Pereira, pp. 39-56.

(2005): Didáctica de la disertación en la enseñanza de la filosofía. Métodos y procedimientos. Bogotá: Cooperativa Editorial Magisterio. Colección Didácticas Magisterio. $100 \mathrm{p}$.

(2007): Disertación en la enseñanza de la filosofía: definición, procedimientos y escritura. Comunicación presentada en el II Seminario internacional didácticas de la filosofía. Biblioteca Piloto. Auditorio Manuel Mejía Vallejo. Medellín. Universidad de Antioquia-Universidad del Valle-Universidad Pedagógica Nacional-Universidad del Rosario. 10, 11, 12 de octubre de 2007. 15 páginas. (En proceso de edición por la Universidad de Antioquia y la Universidad Pedagógica Nacional).

JONNAERT, Philippe (2004): Constructivisme et curricula. Constructivisme : choix contemporaines. Hommage à Ernest Von Glasersfeld. Sous la direction de Philippe Jonnaert et Dominique Masciotra. Québec : Col. Education-Intervention. Editions Presses de l'Université du Québec, pp. 65-120.

KoHAN, Walter; Walkman (Comps.) (2000): Filosofía para niños. Discusiones y propuestas. Buenos Aires: Ediciones Novedades Educativas. 219 p.

LEVENT, Jean-Marc (2001): "La resistencia en la obediencia. Miradas sobre la historia de la enseñanza de la filosofía en Francia (1945-1999)". Suma Cultural. N. ${ }^{\circ} 3$-marzo 2001, pp. 79-98.

PINEDA, Diego Antonio (2004): “¿En qué consiste una educación filosófica?”. En: Germán Vargas Guillen, Luz Gloria Cárdenas Mejía (Editores): Filosofía, pedagogía y enseñanza de la filosofía..Bogotá: Universidad Pedagógica Nacional. Colección: Filosofía y enseñanza de la filosofía -1. Pp. 125-161.

POUCET, Bruno (1996): Histoire de l'enseignement de la philosophie en France dans

l'enseignement secondaire de 1863 á 1965. ANTRT. Thése á la carte. Lille. Vol. 1 y 2. (2001) : «La "classe de philosophie" dans son histoire». Côte Philo. Le journal de l'enseignement de la philosophie. 4, pp. 11-15. 
SÁnCHEz HuerTAS, María José (1996): "La didáctica de la filosofía en Italia”. Diálogo filosófico 36 (1996), pp. 431-454.

SARMIENTO REYES, Juan Carlos (2004): "Estado de la práctica de la investigación y la enseñanza de la filosofía”. En: Germán Vargas Guillen, Luz Gloria Cárdenas Mejía (Editores): Filosofía, pedagogía y enseñanza de la filosofía..Bogotá: Universidad Pedagógica Nacional. Colección: Filosofía y enseñanza de la filosofía -1. Pp. 325-350.

SERRAT, Lisa ( 2001): La classe de philosophie de Saint-Simon à nos jours. These DEA. Université de Montpellier. Recuperado el 14 de agosto de 2008, en: http://pratiquesphilo.free.fr/contribu/contrib52.htm

TozzI, Michel (2004) : "La discussion en éducation et formation. Developper un nouveau champ de recherches". La discussion en éducation et en formation. Un nouveau champ de recherches. Sous la direction de Michel Tozzi et Richard Etienne. Paris : L'Harmattan. Pp. 9-19.

(2005): Approche philosophique et didactique de la philosophie. [En línea]. Recuperado el 30 de julio de 2008, en http.//www.philotozii.com/ ?p=244. (2005a): Place et valeur de la discussion dans les nouvelles pratiques à visée philosophique. [En línea]. Recuperado el 30 de julio de 2008, en http.//www.philotozii.com/ ?p=249.

(2006): La transposition didactique. [En línea]. Recuperado el 12 de abril de 2006, en http://www.crdp-montpellier.fr/ressources/agora/D011050.HTML (2007): "Sobre la didáctica del aprendizaje del filosofar". Diálogo filosófico 68 (2007), pp. 207-215. Traducción de Gabriel Arnaiz.

(2008): «Paradigmas organisateurs de l'ensignement philosophique (Les)». Dizionario di didattica della filosofia. [En línea]. Recuperado el 14 de agosto de 2008, en http://www.filosofiamo.com/dizionario_zoom.aspx?pubblicazione.id=1070

UNESCO (2007): La philosophie. Une Ecole de la Liberté. Enseignement de la philosophie et apprentissage du philosopher. Etat des lieux et regards pour l'avenir. Paris: Ëditions Unesco. 277 p. 